Note d'information

\title{
BILAN DES ACTIONS DU PLAN \\ MIGRATEURS CONCERNANT L'ALOSE FEINTE \\ (ALOSA FALLAX RHODANENSIS) SUR LE BASSIN RHÔNE-MÉDITERRANÉE-CORSE.
}

\author{
I. LEBEL (1), J.Y. MENELLA (1), M. LE CORRE (2)
}

(1) Association Migrateurs Rhône-Méditerranée (MRM), Zl du Port Fluvial, Chemin des Ségonnaux, 13200 ARLES, France.

(2) Laboratoire d'Ecologie Aquatique, INRA, 65 rue de Saint-Brieuc, 35042 RENNES Cedex, France.

\section{RÉSUMÉ}

La mise en place du premier volet du plan migrateurs Rhône-Méditerranée a nécessité tout un ensemble de travaux d'aménagement et d'ingénierie pour améliorer la libre circulation des poissons dans la zone concernée. Cet ensemble d'interventions a été accompagné d'études pour suivre ou optimiser les travaux d'aménagement, ainsi que de mesures réglementaires.

Sur le Rhône et ses affluents, les trois écluses de navigation de Vallabrègues, d'Avignon et de Caderousse ont été aménagées pour permettre la réalisation d'éclusées réservées spécialement au passage des poissons tandis que de nombreux seuils ont été modifiés ou équipés pour favoriser leur franchissement par les aloses.

L'ensemble des études a permis d'abord d'améliorer les connaissances sur la taxinomie, la biologie et l'écologie de l'alose feinte du Rhône. Les résultats ont ainsi mis en évidence une sous-espèce endémique qui renforce l'intérêt d'un programme de restauration des stocks dans une optique de maintien de la biodiversité.

Le suivi de la pêcherie amateur aux engins a permis de préciser la fenêtre optimale de migration des aloses, et les facteurs influant sur la remontée de reproduction des adultes.

Les résultats des campagnes de comptages visuels et d'échosondage ont montré l'efficacité globale des éclusées à poissons en tant que système de franchissement de l'ouvrage. Seul le comptage visuel permettrait d'évaluer le nombre de poissons franchissant l'obstacle, tout en restant assujetti à des contraintes techniques et à des imprécisions inhérentes à cette technique. 
Enfin, l'étude récente de la pêche sportive par carnets de pêche s'avère particulièrement utile dans une analyse qualitative de l'extension de la zone colonisée par les aloses, qui a incontestablement augmenté ces sept dernières années.

En 1998, un bilan scientifique des actions et études menées depuis 1991 a été réalisé pour amorcer la réflexion sur l'efficacité et l'orientation à donner au plan migrateurs pour compléter les objectifs prévus ainsi qu'optimiser et étendre le programme de restauration:

- mettre en place un tableau de bord des indicateurs du potentiel « migrateurs ", des actions de suivi et d'aménagement, afin d'améliorer la lisibilité du programme,

- maximiser et évaluer le stock franchissant le barrage de Vallabrègues,

- améliorer les possibilités de franchissement des obstacles, dès le cours aval,

- intensifier le suivi de la pêche à la ligne,

- identifier par radiopistage le comportement du poisson en aval de Vallabrègues.

A l'issue des sept années d'études et de travaux, l'objectif principal sur le Rhône est d'améliorer les connaissances d'un point de vue quantitatif mais également qualitatif avec l'évaluation du stock d'aloses et du flux migratoire sur le premier obstacle.

Parallèlement, une extension du volet aloses du plan migrateurs aux autres bassins méditerranéens français a été engagée sur l'Aude, l'Hérault, le Vidourle, l'Argens et le Tavignano en Corse.

Mots-clés : alose, plan migrateurs, Rhône-Méditerranée-Corse, aménagements, études, passes à poissons, colonisation.

\title{
BALANCE SHEET OF THE MIGRATORY FISH PROGRAM \\ ACTIONS FOR THE TWAITE SHAD POPULATION \\ (ALOSA FALLAX RHODANENSIS) ON THE RHONE-MEDITERRANEAN-CORSICA BASIN
}

\begin{abstract}
The practical application of the first part of the migratory fish program on the RhoneMediterranean-Corsica basin demanded a whole lot of equipping and engineering to improve the circulation of the fish in the concerned zone. These interventions were coupled with researches to follow up or optimize the different installations, and the different statutory measures.

In the Rhone and its tributaries, the three navigation locks of Vallabregues, Avignon and Caderousse have been arranged to permit special passages for the fish while numerous dams have been adapted or equipped so that the shads can clear them.

Studies as a whole permitted to improve the knowledge of the taxonomy, the biology and the ecology of the twaite shad of the Rhone. The results show the evidence of an endemic subspecies which re-enforces the interest of a stock restoration program to conserve the biodiversity.
\end{abstract}


The monitoring of amateur square fishing net fishery allowed to pinpoint the optimal shad migration period and the different factors influencing the reproduction ascent of the adults.

The results of the visual counting campaigns and that of the echoprobe showed the global efficiency of the fish sluices as a system of clearing the dam. The visual counting was the only mean to evaluate the number of fish passing through the dam, but it nevertheless suffered from constraints and imprecision inherent to its nature in itself.

Finally, recent studies of sport fishing by angling logbook is particularly useful in a qualitative analysis of the extension of the zone colonized by shads, which has undeniably grown these past seven years.

In 1998, a scientific balance sheet of surveys and actions done since 1991 was carried out to begin reflections on the efficiency and the orientation to give to this migratory fish plan to complete the wanted objectives as well as to optimise and enlarge the restoration program :

- install a monitoring board of the migratory fish potential, the different actions of improvement and follow up so as to improve the understanding of the program ;

- maximize and assess the stock going through the Vallabregues dam ;

- improve the possibilities of clearing obstacles as far downstream as possible ;

- intensify the study of angling ;

- identify by radiotracking, the behaviour of the fish downstream from Vallabregues.

After seven years of surveys, the main objective on the Rhone is to improve the quantitative and qualitative knowledge with an evaluation of the number of shads and their migratory flow past the first obstacle.

Concurrently, an extension program of the shad migration plan to other french mediterranean basins has begun on the rivers Aude, Herault, Vidourle, Argens and Tavignano in Corsica.

Key-words : shad, migratory fish plan, Rhone-Mediterranean-Corsica, developments, surveys, fish channels, colonization.

\section{INTRODUCTION : ÉVOLUTION DE L'AIRE DE RÉPARTITION DE L'ALOSE SUR LE BASSIN RHÔNE-MÉDITERRANÉE-CORSE (RMC)}

\section{Répartition historique de l'alose sur le bassin RMC (Figure 1)}

Jusqu'au milieu du XXème siècle, les aloses remontaient le Rhône jusqu'au canal de Savière (lac du Bourget) et un certain nombre de ses affluents : le Gardon, la Durance, la Cèze, l'Ardèche, l'Isère jusqu'à Grenoble, la Saône jusqu'à Auxonne et l'Ain (KREITMANN, 1932 ; PATTEE, 1988 ; ALLARDI et KEITH, 1991). 


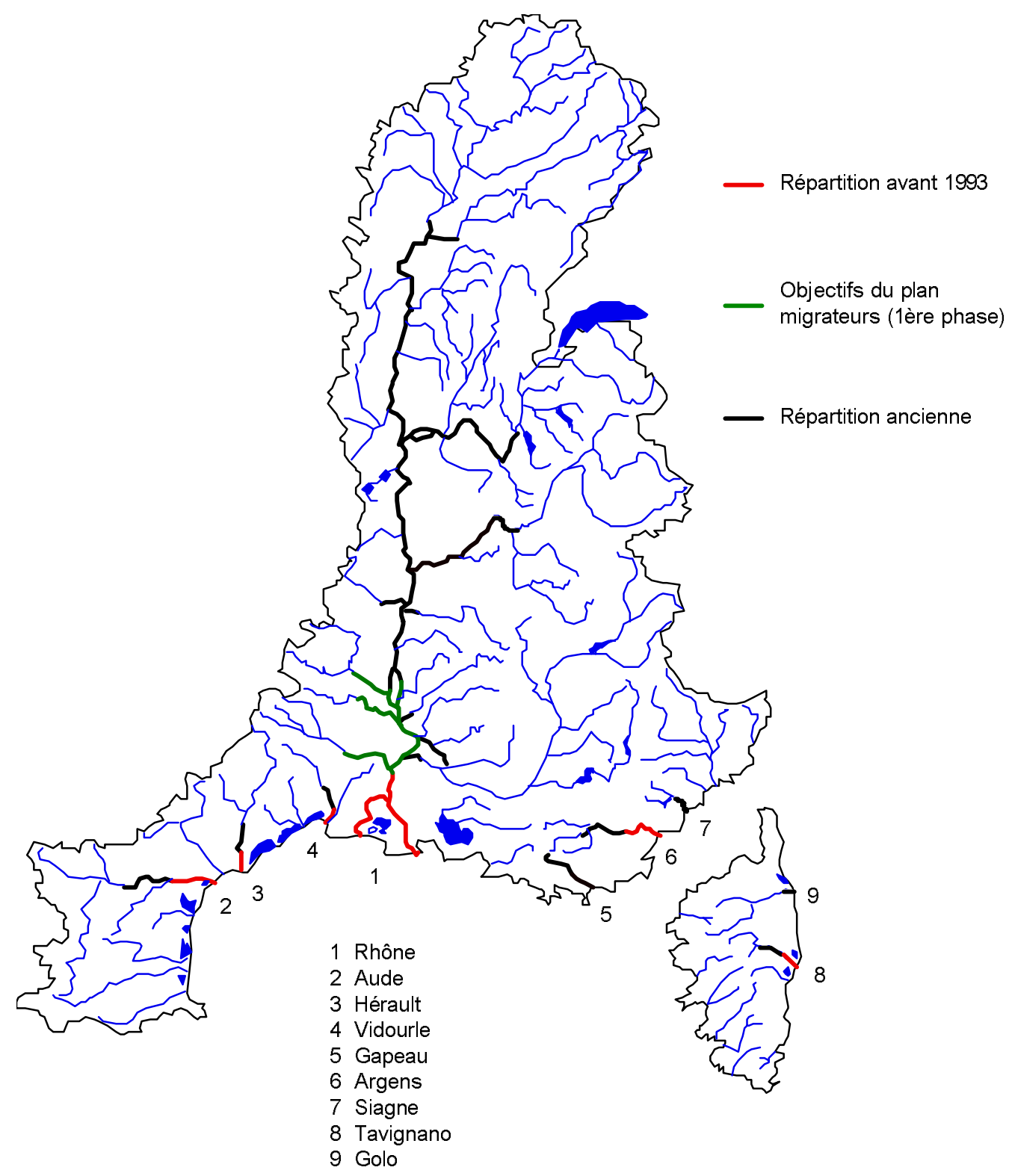

Figure 1

Aire de répartition de l'alose sur le Bassin Rhône-Méditerranée-Corse.

Figure 1

Geographical distribution of shad on the Rhone-Mediterranean-Corsica Basin.

A partir de 1947, l'aire de répartition de l'espèce régresse rapidement sur le Rhône à la suite de la construction du barrage de Donzère-Mondragon qui bloque les aloses quelques kilomètres en amont de l'Ardèche (GALLOIS, 1947 ; PATTEE, 1988). De ce fait, alors que les pêches d'aloses atteignaient 53 tonnes entre Arles et Pont-Saint-Esprit en 1927, elles ne s'élèvent plus qu'à 10 tonnes en 1950 (RAMEYE et al., 1976 ; CASSOULEINS et CASSOU-LEINS, 1981).

Des frayères sont encore signalées entre Beaucaire et Avignon, mais les aloses ne remontent plus guère au-delà de Lyon (ALLARDI et KEITH, 1991). 
La construction du barrage de Beaucaire-Vallabrègues (en 1971), puis plus tard celle d'autres ouvrages, limitera encore l'aire de remontée des aloses. Aussi, en 1988 et 1989, les captures moyennes annuelles d'aloses réalisées par les pêcheurs aux engins ne sont plus que de 6,3 tonnes (CHANGEUX et ZYLBERBLAT, 1993).

L'espèce est cependant signalée sur d'autres fleuves méditerranéens (ALLARDI et KEITH, 1991).

Ainsi, à la fin des années 60, l'alose remontait l'Aude jusqu'à Carcassonne, l'Hérault jusqu'à sa confluence avec le Lergue (environ 50 kilomètres), et le Vidourle jusqu'à l'amont de sa confluence avec la Bénovie (40 à 50 kilomètres de l'embouchure).

De plus, en région Provence-Alpes-Côte d'Azur, l'alose était présente sur la quasitotalité du linéaire du Gapeau, ainsi que sur l'Argens et la Siagne (ANONYME, 2000), et en Corse sur le Tavignano et le Golo (ALMACA, 1988).

Les limites amont de répartition sont liées à la présence d'obstacles physiques (barrages, seuils) qui bloquent la migration des aloses. Mais d'autres facteurs ont conditionné le maintien ou la disparition des populations (ANONYME, 1995) :

- les extractions de granulats qui détruisent les zones de frayères et les zones de grossissement des alevins,

- les bouchons de pollution urbaine, agricole et industrielle qui bloquent les aloses au niveau des estuaires, celles qui parviennent à les franchir prenant du retard dans leur migration (décalage avec les conditions favorables à la ponte notamment),

- la construction de centrales hydro-électriques qui provoque, par les pompages nécessaires à leur fonctionnement, une importante mortalité des juvéniles dévalants,

- le passage dans les turbines des micro-centrales hydro-électriques qui entraîne une mortalité non négligeable d'adultes et de juvéniles en dévalaison.

\section{Aire de répartition au démarrage du plan migrateurs (Figure 1)}

En 1993, au démarrage du plan migrateurs Rhône-Méditerranée, l'alose n'était plus présente sur le Rhône qu'en aval du barrage de Vallabrègues (QUIGNARD, 1978; KEINER, 1985 ; ALLARDI et KEITH, 1991 ; CHANGEUX et PONT, 1995). En effet, celui-ci constitue le premier obstacle à la migration des aloses sur le Rhône; de plus, il barre l'accès aux affluents.

Sur les fleuves côtiers du Languedoc-Roussillon (Aude, Hérault, Vidourle), l'aire de répartition des aloses a considérablement diminué et se trouve limitée à la partie aval des cours d'eau, jusqu'au premier obstacle (seuil ou barrage). De plus, les niveaux de population semblent très faibles (PORCHER, 1992).

De même, sur les fleuves côtiers de la région Provence-Alpes-Côte d'Azur, l'aire de colonisation de l'alose a été considérablement réduite du fait des aménagements des cours d'eau. L'espèce est encore présente sur l'Argens (LANGON, 1999a). En revanche, le doute persiste quant à la présence de l'alose sur le Gapeau et sur la Siagne.

En Corse, les aloses sont présentes sur le Tavignano jusqu'à la micro-centrale de Cardiccia (LANGON, 1999b). Leur présence sur le Golo n'est pas confirmée. 


\section{OBJECTIFS DU PLAN MIGRATEURS RHÔNE-MÉDITERRANÉE}

\section{Sur le Rhône et son bassin versant}

Sur le bassin du Rhône, en aval de la confluence avec l'Ardèche, le Contrat "Retour aux Sources » prévoyait deux axes de travail (PORCHER, 1992):

- la restauration de la libre circulation avec l'aménagement des écluses de navigation des grands barrages hydro-électriques de Vallabrègues, d'Avignon et de Caderousse ainsi que de sept seuils sur le Gardon (premier affluent de rive droite),

- l'évaluation du stock par quantification des passages à l'écluse de Vallabrègues et enquête sur les captures réalisées par la pêcherie.

Cependant, suite aux propositions du Schéma de Vocation Piscicole du Rhône (ANONYME, 1991), ces objectifs ont été élargis, et le plan migrateurs doit assurer :

- le rétablissement de la circulation de l'alose sur l'axe rhodanien et ses affluents en aval de la confluence avec l'Ardèche, ainsi que la protection des frayères à aloses existantes et potentielles de cette zone,

- la restauration de la population d'anguilles jusqu'à Lyon.

Après la validation de ces propositions par la Commission de Bassin en 1991, un atelier " migrateurs » a été créé afin de définir techniquement et financièrement le plan migrateurs Rhône-Méditerranée, tant en termes d'études et de communication qu'en matière d'investissements.

Cet atelier «migrateurs » a mené un certain nombre d'études et de travaux et n'a retenu pour la première phase du programme que l'objectif concernant les aloses. Puis il a découpé la zone d'action en deux volets :

- le volet A comprenant l'axe de navigation du Rhône et ses affluents de rive droite (Gardon, Cèze et Ardèche),

- le volet B constitué par le Bras du Rhône d'Avignon ainsi que par les affluents de rive gauche (Durance, Ouvèze et Aygues).

Les travaux du volet $A$ ont été engagés en premier dès 1993 (Figure 2), tandis que la définition du volet $B$ devait être précisée après analyse de potentialités de son réseau pour l'alose.

Sur le bassin du Rhône, l'Association Migrateurs Rhône-Méditerranée a été créée en 1993, afin de gérer et de coordonner le plan migrateurs Rhône-Méditerranée, tant sur le plan technique que financier, tout en assurant l'information et la sensibilisation du public.

\section{Sur les fleuves côtiers du bassin RMC}

Sur le Bassin RMC, le Contrat "Retour aux Sources » prévoyait, sur la période 1991-1995, des actions sur l'Aude, l'Hérault pour la restauration des populations d'aloses (PORCHER, 1992) :

- sur l'Aude (DESJAMES, 1987), trois thèmes de travail avaient été identifiés :

* la restauration de la libre circulation des poissons avec la construction d'une passe migratoire sur le seuil de Moussoulens, 
* une évaluation du stock lors du contrôle de la migration dans cette passe,

* la restauration du milieu en révisant le débit réservé à l'aval de Moussoulens afin de réhabiliter les zones de croissance des alosons.

- sur l'Hérault (CRUZ, 1991), il était prévu de :

* restaurer la libre circulation avec l'aménagement des seuils d'Agde, de Florensac et de Saint-Thibéry,

* d'évaluer le stock par la quantification des passages et l'étude des rythmes de migration par piégeage ou observations vidéo dans les passes migratoires.

Sur les autres cours d'eau du bassin méditerranéen, le plan de gestion des poissons migrateurs 1995-1999 du Comité de Gestion des Poissons Migrateurs (COGEPOMI) préconisait la mise en œuvre de mesures spécifiques, ainsi que la synthèse préalable des diagnostics et propositions des schémas piscicoles (ANONYME, 1995), avec en particulier :

- l'évolution de la qualité du milieu physique et chimique (effets retardés de l'extraction abusive de granulats, de l'urbanisme et de l'eutrophisation),

- les pratiques et réglementations applicables en zone estuarienne, propositions d'harmonisation des limites de domaine, surveillance des sites et police,

- l'état et la structure des stocks d'anguilles, d'aloses et autres migrateurs amphihalins, ainsi que l'aménagement des obstacles pour le passage des poissons.

\section{RÉSULTATS ET BILAN DES ACTIONS DU PLAN MIGRATEURS RHÔNE- MÉDITERRANÉE}

\section{Sur le Rhône et son bassin versant}

Aménagements et travaux réalisés

Eclusées à poissons (Figure 2)

Le fonctionnement de l'écluse en tant que passe à poissons avait été envisagé dès la construction de l'ouvrage de Beaucaire-Vallabrègues.

Un protocole a pu être mis au point (RUCHON et al., 1994 ; BECHU et al., 1995 ; GENDRE, 1997a ; GENDRE, 1997b ; GENDRE, 1997c), tenant compte des contraintes techniques inhérentes à la navigation et des exigences des aloses (Figure 3).

Ainsi, de la mi-mars à la fin juin, le nombre d'éclusées à poissons réalisées chaque jour est de deux (au minimum) à cinq (en période optimale de migration), le fonctionnement de l'écluse de Vallabrègues en tant que passe à poissons ayant été automatisé en 1996. 


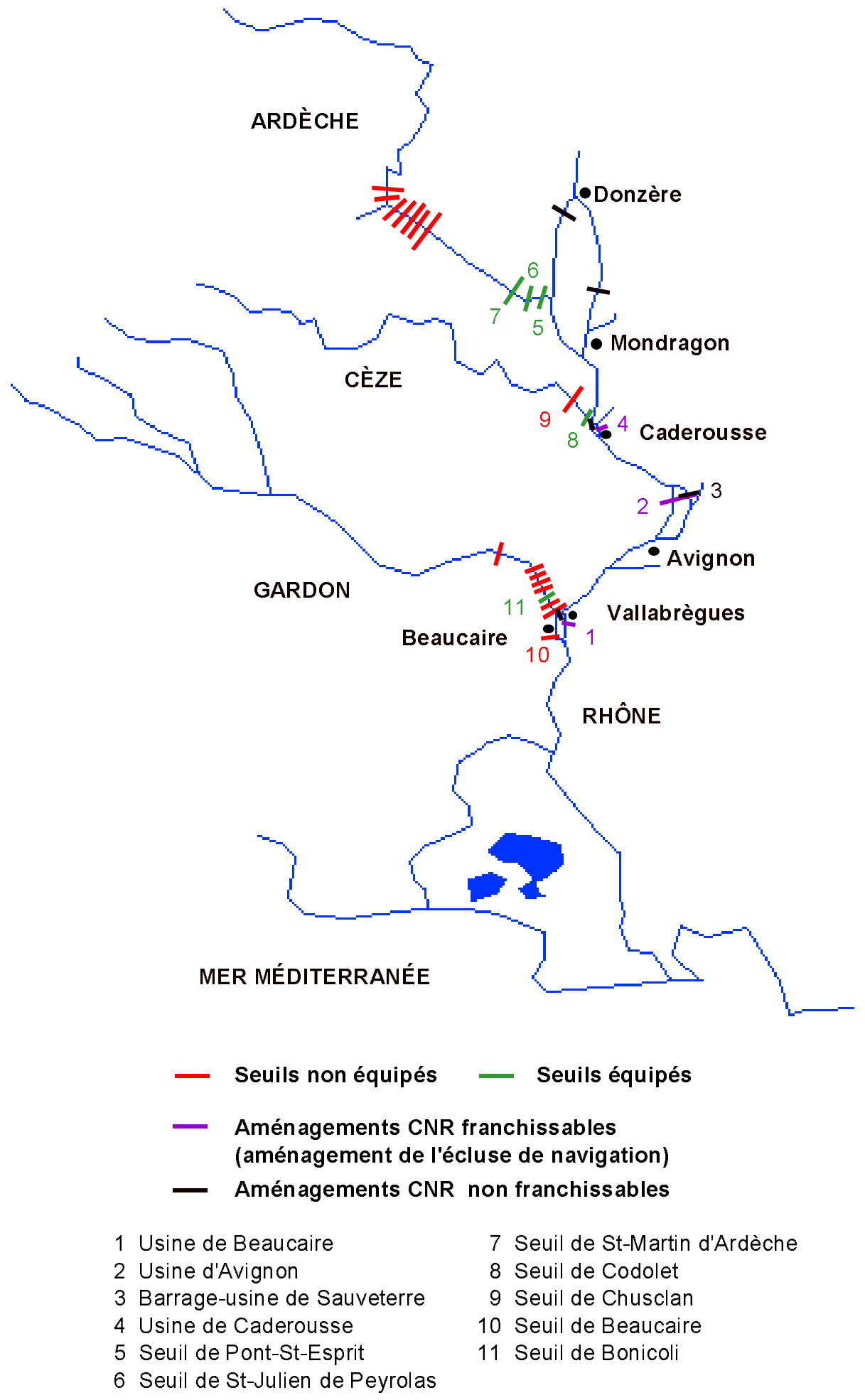

\section{Figure 2}

Aménagements sur le bassin du Rhône.

\section{Figure 2}

Equipments on the Rhone basin. 

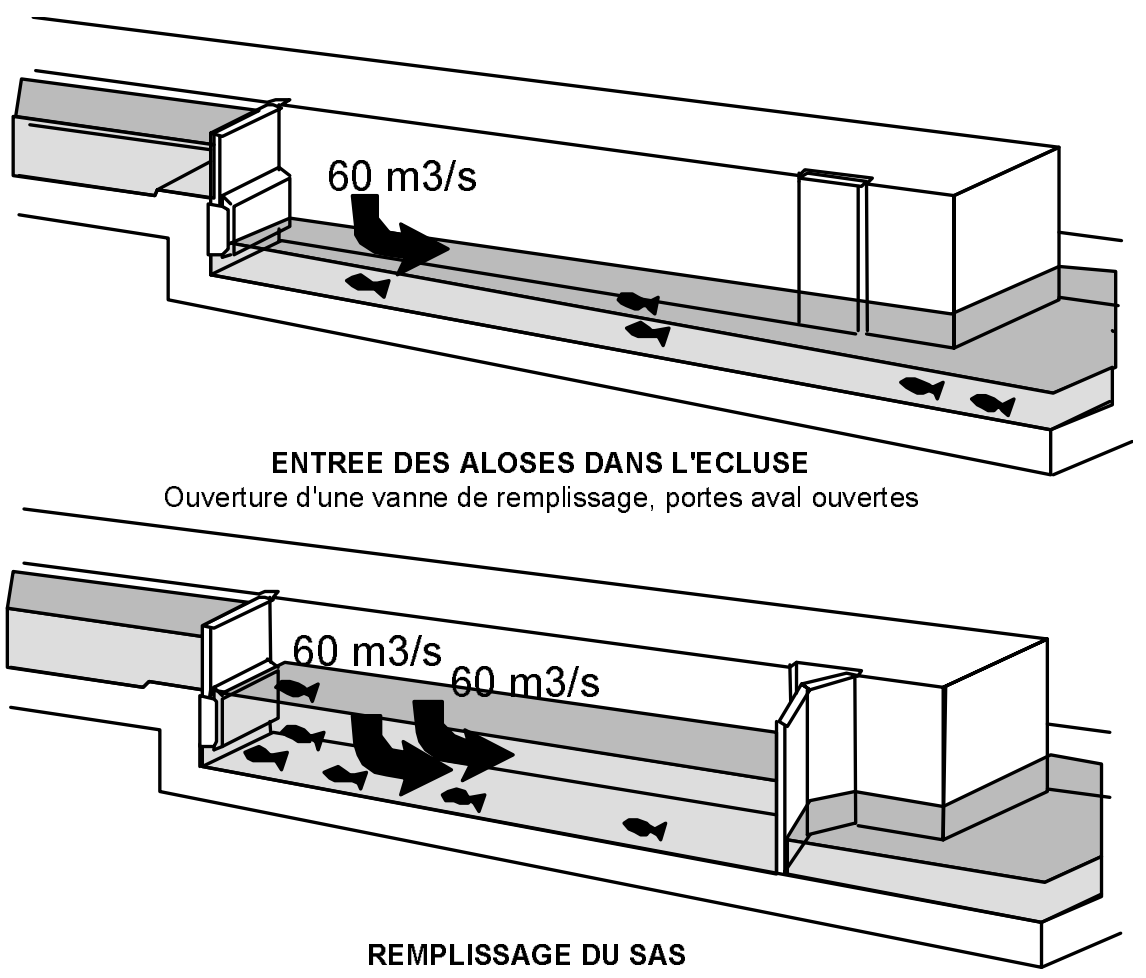

Fermeture des portes aval et ouverture des deux vannes de remplissage

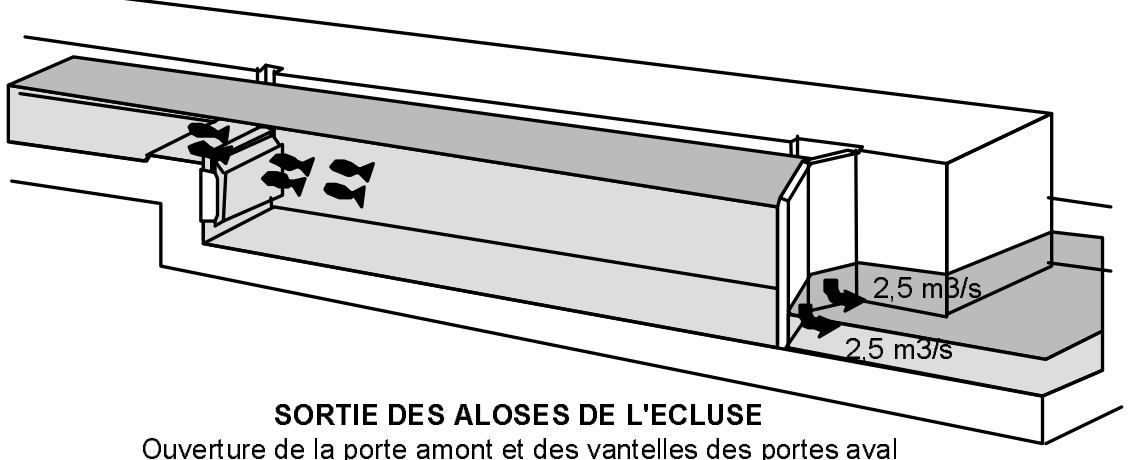

Ouverture de la porte amont et des vantelles des portes aval

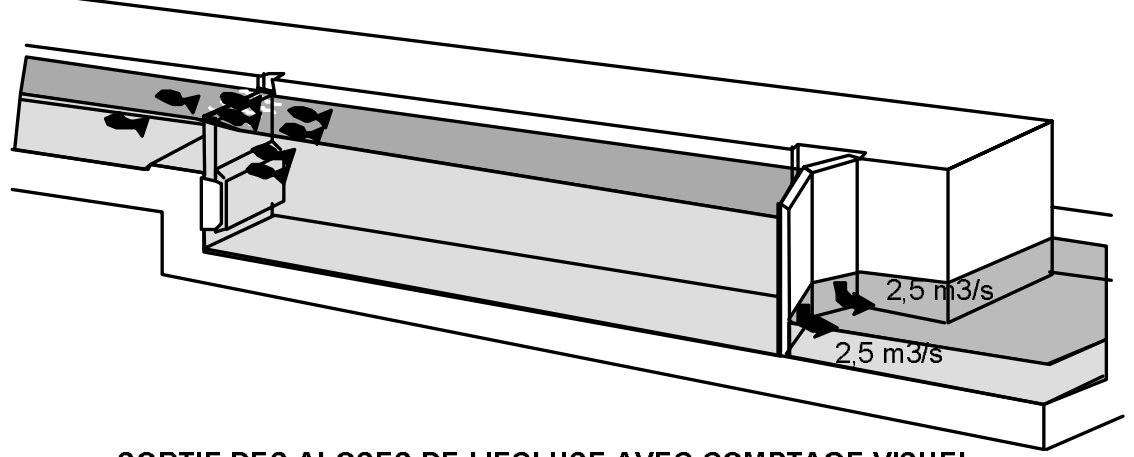

SORTIE DES ALOSES DE L'ECLUSE AVEC COMPTAGE VISUEL

Ouverture partielle de la porte amont et ouverture des vantelles des portes aval

\section{Figure 3}

Fonctionnement des écluses en passes à poissons.

\section{Figure 3}

Functioning of locks as fish ladders. 
De la même manière, les écluses d'Avignon et de Caderousse ont été aménagées pour permettre la réalisation d'éclusées à poissons et leur fonctionnement a été automatisé respectivement en 1998 et 1999.

\section{Passes à poissons (Figure 2)}

Sur l'Ardèche, les seuils de Pont-Saint-Esprit et de Saint-Julien de Peyrolas ont été rendus franchissables par conception et une passe à poissons a été construite sur le seuil de Saint-Martin d'Ardèche.

Sur la Cèze, une passe à poissons a été construite sur le seuil de Codolet et la construction de celle du seuil de Chusclan est prévue pour 2002.

Sur le Rhône court-circuité de Beaucaire, la passe à poissons du seuil de Beaucaire (immédiatement en aval de la confluence avec le Gardon) sera construite en 2001.

Sur le Gardon, la passe du seuil de Bonicoli a été achevée en 1999. De plus, une étude globale sur les autres seuils (conception, plan et coût des passes à poissons) est en cours et doit permettre la programmation des travaux.

\section{Acquisition de connaissances sur l'alose du Rhône}

Les études réalisées de 1994 à 1998 ont permis d'améliorer les connaissances sur la taxinomie, la biologie et l'écologie de l'alose feinte du Rhône (LE CORRE et al., 1996 ; LE CORRE et al., 1998a).

\section{Statut de l'Alose du Rhône}

Une première étude sur les aloses de la Méditerranée française (DOUCHEMENT, 1981) ne donnait pas de conclusion claire sur leur statut génétique.

De ce fait, une nouvelle étude a été réalisée (LE CORRE et al., 1996 ; LE CORRE et al., 1998a ; LE CORRE, 1999).

Ainsi seul le taxon Alosa fallax rhodanensis (Roule, 1924) est présent.

Les caractéristiques de ce taxon, alliées à un phénomène d'itéroparité important, le rattachent à l'alose feinte, bien que ses caractères biométriques (taille, poids, forte croissance) et ses longues distances de migration à l'intérieur du fleuve (avant les aménagements) le rapprochent de la grande alose (Tableau I).

Toutefois, si les populations méditerranéennes étudiées (Rhône et Aude, auxquelles s'ajoutent les quelques individus capturés sur l'Hérault) sont génétiquement très proches entre elles, elles représentent un groupe particulier se dégageant nettement des populations d'aloses feintes étudiées sur la façade Atlantique, en France et au Portugal (ALEXANDRINO, 1996 ; LE CORRE, 1999).

Ainsi, les résultats des premiers travaux génétiques menés sur les populations d'aloses feintes de Méditerranée ne seraient pas en désaccord avec l'attribution du rang de sous-espèce à ce taxon (LE CORRE, 1999 ; LE CORRE et al., données non publiées). Ces résultats restent cependant à confirmer par l'étude d'un plus grand nombre de populations méditerranéennes en France, mais aussi en Espagne et en Italie. 


\section{Tableau I}

Principales caractéristiques démographiques des aloses échantillonnées sur le Rhône.

Table I

Main demographic characteristics of the shads sampled in the Rhone.

\begin{tabular}{|c|c|c|c|c|c|c|c|}
\hline Année & Sexe & 1994 & 1995 & $1994 / 95$ & 1996 & 1997 & 1998 \\
\hline \multirow{2}{*}{$\begin{array}{c}\text { Spectre de } \\
\text { classes d'âge }\end{array}$} & Mâles & 3 à 7 ans & 3 à 8 ans & & 3 à 7 ans & 3 à 7 ans & 3 à 5 ans \\
\hline & Femelles & 3 à 8 ans & 3 à 7 ans & & 3 à 8 ans & 3 à 7 ans & 3 à 7 ans \\
\hline \multirow{3}{*}{$\begin{array}{l}\text { Age moyen } \\
\text { de } \\
\text { remontée }\end{array}$} & Population & 4,5 & 5,3 & & & & \\
\hline & Mâles & 4,1 & 4,7 & 4,3 & 5,1 & 4,3 & \\
\hline & Femelles & 5 & 5,5 & 5,4 & 5,9 & 4,8 & \\
\hline \multirow{2}{*}{$\begin{array}{l}\text { Age moyen de première } \\
\text { reproduction }\end{array}$} & Mâles & 3,5 & 4 & 3,7 & 4,7 & 3,7 & 4 \\
\hline & Femelles & 4 & 4,4 & 4,3 & 5,1 & 4,2 & 4,6 \\
\hline \multirow{3}{*}{$\begin{array}{l}\text { Pourcentage d'aloses } \\
\text { s'étant déjà reproduit } \\
\text { au moins une fois }\end{array}$} & Population & \multicolumn{2}{|c|}{$49,3 \%$} & & & & $25,3 \%$ \\
\hline & Mâles & $35,1 \%$ & $39,6 \%$ & & $33 \%$ & $40 \%$ & $11,6 \%$ \\
\hline & Femelles & $54,7 \%$ & $57,4 \%$ & & $62 \%$ & $38 \%$ & $13,7 \%$ \\
\hline \multirow{2}{*}{$\begin{array}{l}\text { Longueur totale } \\
\text { moyenne (mm) }\end{array}$} & Mâles & & & & 425 & 451 & 436 \\
\hline & Femelles & & & & 523 & 524 & 516 \\
\hline \multirow{2}{*}{$\begin{array}{l}\text { Longueur à la fourche } \\
\text { moyenne }(\mathrm{mm})\end{array}$} & Mâles & 376 & 413 & & 373 & 396 & 385 \\
\hline & Femelles & 465 & 478 & & 461 & 464 & 462 \\
\hline \multirow{2}{*}{$\begin{array}{c}\text { Poids } \\
\text { moyen }(g)\end{array}$} & Mâles & 672 & 914 & & 606 & 948 & \\
\hline & Femelles & 1352 & 1526 & & 1290 & 1599 & \\
\hline
\end{tabular}

II n'en demeure pas moins que l'alose feinte du Rhône a pleinement sa place dans les programmes d'études concernant la connaissance et le maintien de la biodiversité mis en place récemment en Europe (programme biodiversitas) et à l'échelon national (programme d'action pour la diversité biologique de la flore et de la faune sauvages).

Fenêtre de migration des aloses

Le suivi de la pêcherie amateur aux engins de 1995 à 1998 a permis de caractériser la migration des aloses, et les facteurs influant sur la migration de reproduction des adultes (température, débit), en montrant notamment l'impact négatif du barrage qui crée un retard important à la migration des aloses (BARTHELEMY, 1997 ; BARTHELEMY et MAZENS, 1997 ; LEBEL et al., 1997 ; PINON, 1998).

La fenêtre optimale de migration se situe de la mi-avril à la mi-mai et reste assez semblable au cours de la saison de remontée quelle que soit l'année.

\section{Franchissement des obstacles}

L'efficacité globale des éclusées à poissons en tant que système de franchissement de l'ouvrage pour le poisson a été montrée au cours des campagnes de comptages visuels (Tableau II) (RUCHON et al., 1994 ; GENDRE, 1997a, 1997b ; LEBEL et al., 1997 ; LEBEL et al., 1999 ; MAZENS et CORRAO, 1997 ; BECHU et al., 1995 ; JOLIMAITRE, 1992), et d'échosondage (JOUFFRE, 1997 ; GUILLARD et al., 1996 ; GUILLARD et COLON, 1997 ; GUILLARD et VIDAL, 1998 ; GUILLARD et al., 1998). 


\section{Tableau II}

Résultats des campagnes de comptages visuels de 1991 à 1999.

\section{Table II}

Results of the visual counting campaigns from 1991 to 1999.

\begin{tabular}{|c|c|c|c|c|c|r|}
\hline Année & $\begin{array}{c}\text { Période de } \\
\text { suivi }\end{array}$ & $\begin{array}{c}\text { Nombre } \\
\text { d'éclusées } \\
\text { spécifiques }\end{array}$ & $\begin{array}{c}\text { Nombre } \\
\text { d'aloses } \\
\text { comptées }\end{array}$ & $\begin{array}{c}\text { Nombre de } \\
\text { comptages } \\
\text { pratiqués }\end{array}$ & $\begin{array}{c}\text { Nombre moyen } \\
\text { d'aloses } \\
\text { comptées }\end{array}$ & $\begin{array}{c}\text { Pic de } \\
\text { franchissement à } \\
\text { l'écluse }\end{array}$ \\
\hline $\mathbf{1 9 9 1}$ & $14 / 05-22 / 05$ & 20 & 3324 & 20 & 166,2 & 21 mai $(42,4 \%)$ \\
\hline 1992 & $22 / 04-20 / 05$ & 49 & 10543 & 49 & 215,1 & 14 mai $(32,5 \%)$ \\
\hline 1993 & $03 / 05-27 / 05$ & 23 & 1912 & 10 & 191,2 & $17-19$ mai $(62,4 \%)$ \\
\hline 1994 & $05 / 05-23 / 06$ & 40 & 4090 & 40 & 102,3 & 09 mai $(22,4 \%)$ \\
\hline 1995 & $07 / 04-10 / 07$ & 55 & 308 & 55 & 5,6 & 13 juin $(27,7 \%)$ \\
\hline 1996 & $14 / 05-24 / 06$ & 101 & 980 & 12 & 81,7 & 14 mai $(85,9 \%)$ \\
\hline 1997 & $26 / 03-24 / 06$ & 187 & 3710 & 15 & 247,3 & 20 mai $(86,2 \%)$ \\
\hline 1998 & $04 / 04-29 / 06$ & 142 & \multicolumn{5}{|c|}{ Pas de comptage réalisé } \\
\hline 1999 & $15 / 03-30 / 06$ & 169 & \multicolumn{5}{|c|}{ Pas de comptage réalisé } \\
\hline
\end{tabular}

Les deux premières campagnes s'inscrivent dans le cadre des études préliminaires et correspondent à la définition et la mise au point de la méthode de comptage, notamment l'attrait aval et l'attrait amont avec comptage. migration.

En 1995, les conditions hydrologiques exceptionnelles semblent avoir bloqué la

La campagne 1996 a démarré le 14 mai soit le jour du pic présumé, les résultats sont donc à considérer avec prudence.

En 1997, le nombre de comptages pratiqués a été fortement réduit suite à une décision de la Compagnie Nationale du Rhône (CNR) qui a limité leur nombre à 10-15 au maximum. Les gestionnaires ont en effet estimé que la porte amont de l'écluse n'était pas adaptée à un comptage intensif et que les coûts de comptage étaient trop élevés.

En 1998 et 1999, aucun comptage visuel n'a pu être réalisé à l'écluse de BeaucaireVallabrègues en raison de travaux sur la porte amont.

Les résultats attestent de l'efficacité globale du dispositif d'éclusées à poissons. De plus, chaque année, le pic d'observation des aloses à l'écluse de navigation a lieu autour de la mi-mai.

Les informations sur la quantité d'aloses transitant vers l'amont du barrage restent toutefois limitées puisque aucune donnée n'est disponible sur les éclusées pour la circulation fluviale (LEBEL et al., 1997). Elles permettent néanmoins d'appréhender le comportement de franchissement des obstacles par les aloses.

\section{Connaissance de la reproduction}

Des études de repérage des frayères potentielles et actives et de suivi de la reproduction ont été réalisées de 1993 à 1999 (LEGAULT, 1993; LADET, 1996; GENDRE et al., 1997b ; MAZENS, 1997 ; CHAMPALBERT, 1998; ROURE, 1997 ; GENOUD, 1996 ; LE CORRE et al., 1997 ; ROURE et al., 1997 ; GENOUD, 1997 ; ROURE et SABATIER, 1998 ; GENOUD, 1999a ; GENOUD, 1999b). 
L'observation directe d'une activité de reproduction (bulls) reste un indice de colonisation d'un site par les aloses. Elle permet de conclure sur l'efficacité des aménagements réalisés en aval de la zone de reproduction.

Cependant, l'utilisation de cette méthode paraît limitée pour plusieurs raisons :

- la difficulté de connaître la surface réelle de la zone de frai fréquentée par les géniteurs ; en effet, cette dernière peut varier dans l'espace et dans le temps au cours d'une même année en raison des variations journalières importantes du débit du cours d'eau qui modifient leur typologie et donc le choix de l'emplacement des géniteurs ; de plus, la répartition spatiale des géniteurs en période de reproduction est en partie liée aux conditions de migration sur le bassin (indépendamment des conditions de libre circulation) et aux caractéristiques annuelles du stock (abondance, structure d'âge et de taille),

- la difficulté, liée en partie à ce qui vient d'être évoqué, de l'utilisation du comptage du nombre de bulls pour donner un niveau de fonctionnalité des frayères.

En dépit de ces inconvénients majeurs, si le suivi de la reproduction et la méthode de comptage des bulls sur le Rhône lui-même et ses affluents de grande taille s'avèrent être inefficaces ou presque, il n'en est pas de même sur des cours d'eau de plus petite taille. Il est alors possible à l'aide d'une méthode de suivi simplifiée dans l'espace et dans le temps (visite de frayères ayant été considérées comme potentielles et à nouveau accessibles lors de la période maximale de reproduction) de mettre au point un indice de fonctionnalité des frayères (LEBEL et al., 1999).

Par ailleurs, les études effectuées sur la reproduction n'ont pas permis de caractériser la typologie des frayères d'aloses sur le bassin, si bien que la reconnaissance de frayères potentielles est toujours faite à partir de celle établie pour la grande alose sur des fleuves atlantiques de plus petite taille (LEBEL et al., 1999).

\section{Evolution de la zone colonisée}

L'évolution de l'aire de répartition des aloses sur le bassin du Rhône a été évaluée par le suivi de la pêche à la ligne (LEBEL et al., 1997 ; PINON, 1998 ; LEBEL, 1999), l'étude de la reproduction sur quelques frayères identifiées du Rhône et de ses affluents ainsi que par la tentative de suivi de la dévalaison des alosons (MENELLA et TABARDEL, 1994 ; TABARDEL et al., 1995 ; GENDRE et al., 1997a).

Au terme de 8 années de suivi (1991-1999), l'aire de colonisation des aloses a incontestablement augmenté. En effet, au milieu de la décennie 1970, il y avait très peu d'aloses en amont de Vallabrègues (RAMEYE et al., 1976), alors que le suivi de la pêche à la ligne fait apparaître au fur et à mesure des années une augmentation significative du nombre des captures de plus en plus loin en amont du bassin versant. Ainsi, des captures ont été signalées jusqu'à 163 kilomètres de l'embouchure à Chateauneuf du Rhône et dans l'Ardèche (Figure 4) (LEBEL et al., 1997 ; LEBEL et al., 1999 ; LEBEL, 1999 ; PINON, 1998).

De plus, la présence d'alosons (MENELLA et TABARDEL, 1994 ; TABARDEL et al., 1995 ) et d'aloses adultes (MENELLA, comm. pers.) en dévalaison bloquées au niveau des turbines des usines de Sauveterre et de Caderousse, confirment la colonisation par la population de la zone de reproduction située en amont de l'ouvrage de Vallabrègues (frayères de l'Ardèche, des Rhône court-circuité de Donzère-Mondragon et de Caderousse). 


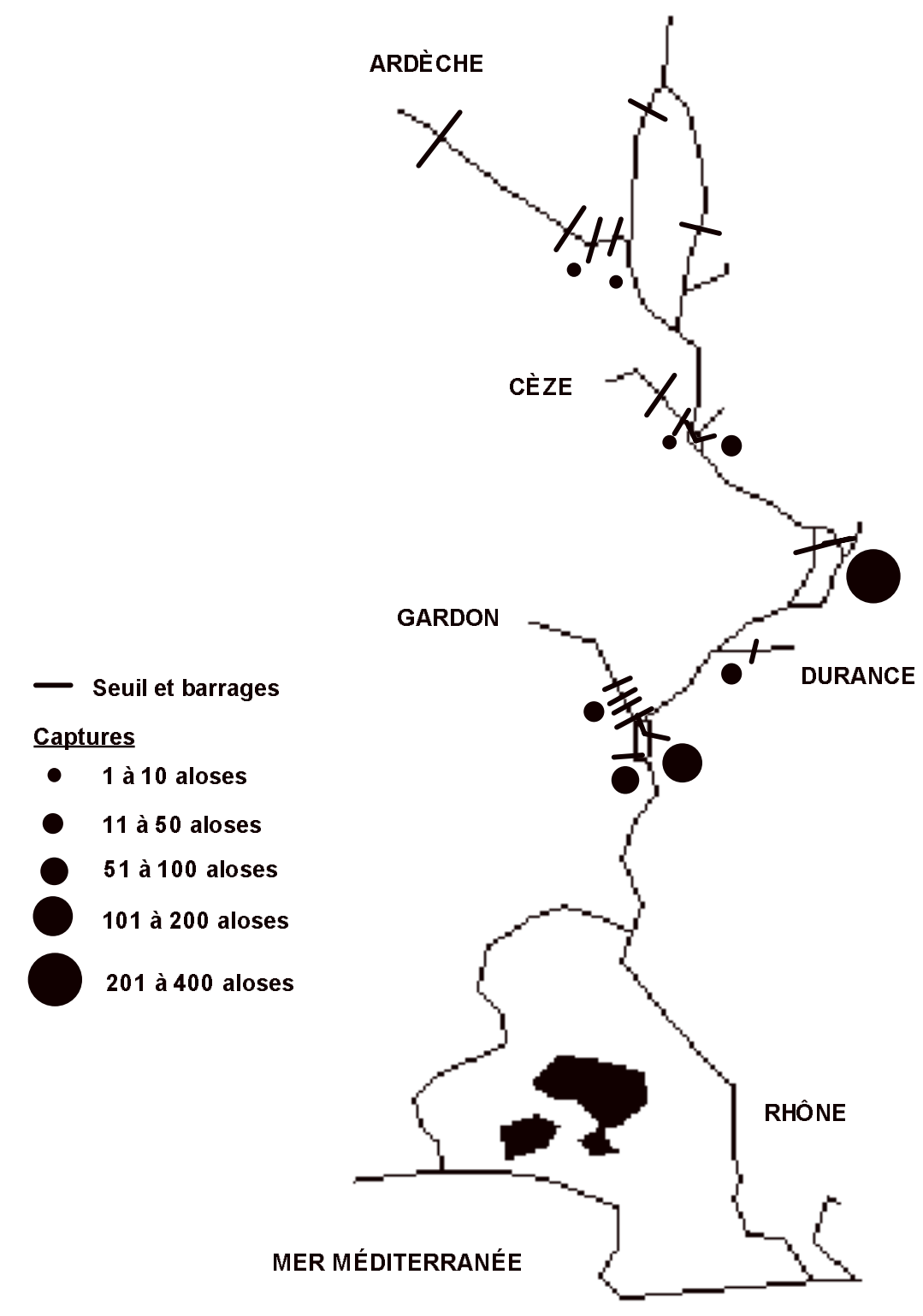

Figure 4

Répartition des captures de la pêche à la ligne sur le bassin du Rhône en 1998.

Figure 4

Distribution of shad catches made by anglers on the Rhone basin in 1998. 
- Seuil et barrages

\section{Captures}

- 1 à 10 aloses

- 11 à 50 aloses

51 à 100 aloses

101 à 200 aloses

201 à 400 aloses

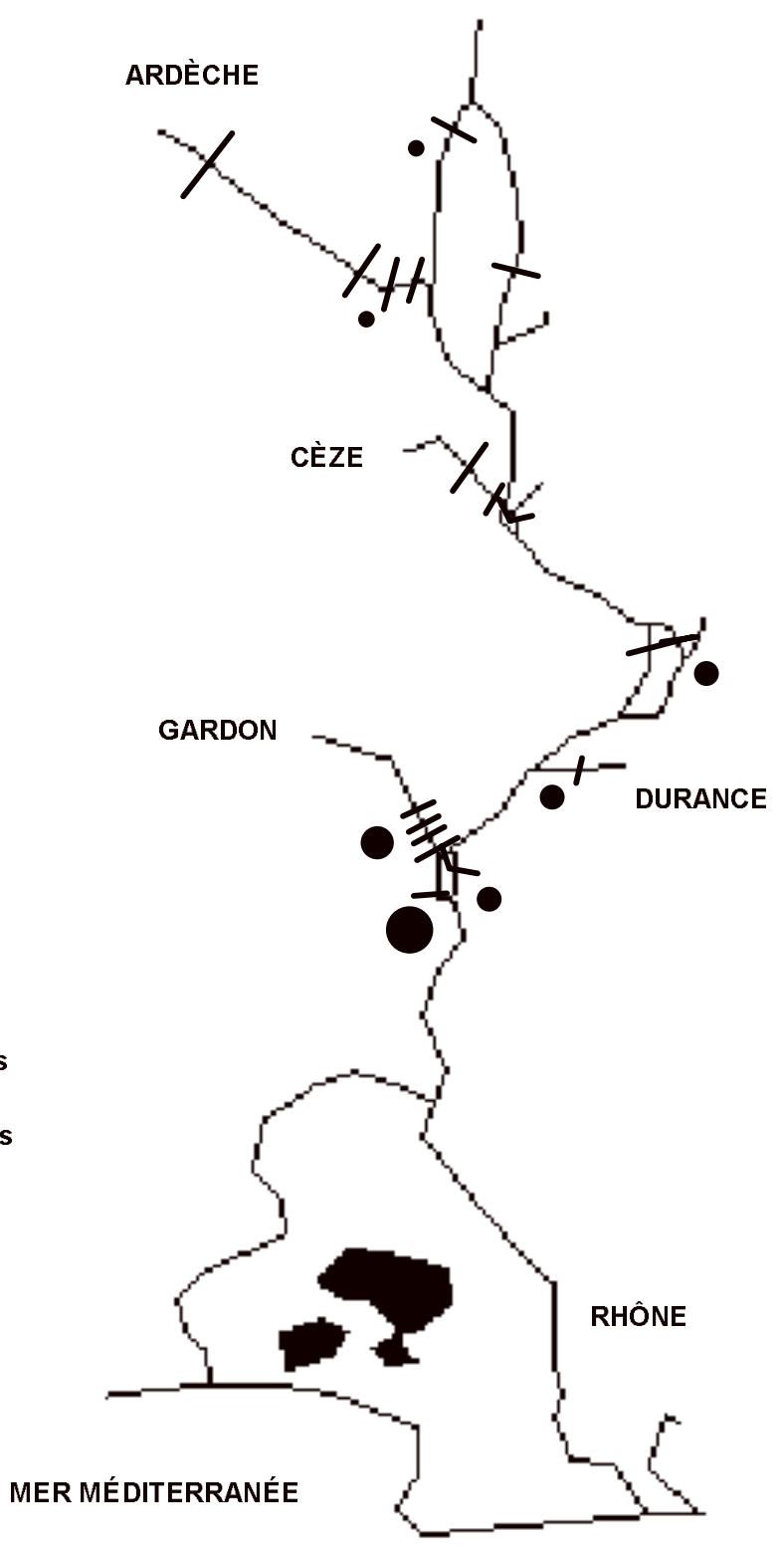

Figure 5

Répartition des captures de la pêche à la ligne sur le bassin du Rhône en 1999.

Figure 5

Distribution of shad catches made by anglers on the Rhone basin in 1999. 
En termes qualitatifs, les mesures spécifiques mises en œuvre (éclusées à poissons, aménagement des cours d'eau, méthodes d'études et de suivi) ont donc permis d'atteindre l'objectif du retour de l'alose sur l'Ardèche.

Cependant, la zone effectivement colonisée une année donnée reste fortement liée aux facteurs du milieu et notamment aux conditions hydrologiques du Rhône et de ses affluents.

En effet, la répartition des captures à la ligne en 1999 (et donc la répartition globale des aloses sur le bassin) a été différente de celle qui a été observée en 1998, et ce principalement en raison du débit du Rhône très différent (Figures 4 et 5) (LEBEL, 1999).

Le débit du Rhône élevé à Beaucaire a engendré un déversement au barrage de retenue qui a favorisé le noyage et donc le franchissement des obstacles du bras courtcircuité et du Gardon. Des aloses ont en effet été capturées en quantités importantes sur le Gardon (Figure 6).

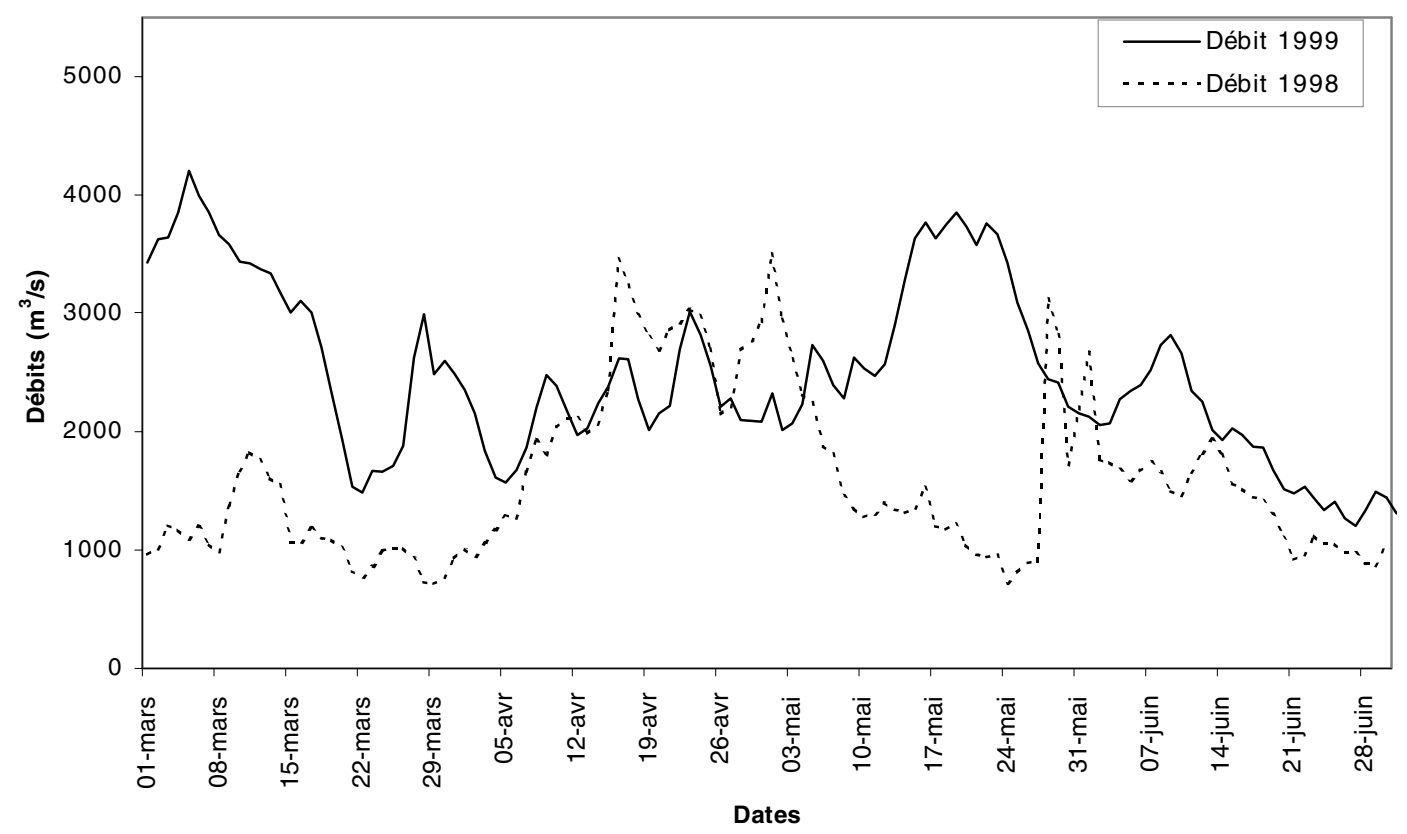

\section{Figure 6}

Débits du Rhône à Beaucaire en 1998 et 1999.

\section{Figure 6}

Downflowes of the Rhone at Beaucaire in 1998 et 1999.

Par ailleurs, en termes quantitatifs, on ne dispose actuellement d'aucune estimation fiable du niveau d'abondance du stock et donc de son évolution depuis la mise en œuvre du plan migrateurs Rhône-Méditerranée (LEBEL et al., 1999).

Tout au plus peut-on dire que le stock ne semble pas résiduel dans la mesure où il supporte une exploitation globale (pêche aux engins et à la ligne) qui se maintient entre 9 et 12 tonnes par an (LEBEL et al., 1999).

De plus, la diminution des captures de la pêche aux carrelets semble indiquer que la fraction disponible pour l'exploitation par cette pêcherie décroît. Cette diminution semble 
due pour partie à des variations annuelles d'abondance (elles peuvent aller de 1 à 100) et de répartition spatiale dans le bassin (en 1995, le débit élevé du Rhône a probablement limité la migration), et pour partie aux mesures mises en place pour augmenter le passage des aloses à Vallabrègues (LEBEL et al., 1999).

Actuellement la seule mesure d'abondance dont on dispose est le nombre d'individus franchissant le barrage de Vallabrègues. Cependant les résultats obtenus à partir de la méthode utilisée restent préliminaires et demandent à être approfondis par une augmentation des comptages visuels d'aloses aux périodes de forte migration (LEBEL et al., 1999).

\section{Sur les autres fleuves côtiers du bassin R.M.C.}

Aude (DESJAMES, 1987)

Après une longue période d'attente pour se mettre en conformité vis-à-vis du classement à migrateurs du cours d'eau selon l'article L.232-6 du Code Rural, Voies Navigables de France (VNF), gestionnaire du seuil de Moussoulens, a finalement décidé son aménagement avec une passe à poissons suite à l'intervention du Conseil Supérieur de la Pêche (CSP) et d'associations locales de protection de la nature auprès de la Préfecture et des services de l'Etat.

Les travaux de construction de la passe à poissons ont démarré pendant l'été 1999 mais elle a été détruite par une violente crue automnale avant qu'elle ne soit achevée. Cette passe devrait donc être reconstruite en 2001 pour être opérationnelle pour la migration 2002.

En conséquence, l'évaluation du stock et la restauration du milieu n'ont pas encore pu être entrepris. De plus, des problèmes de circulation piscicole demeurent sur ce fleuve en raison de l'existence de plusieurs autres seuils :

- en aval du barrage de Moussoulens, le seuil anti-sel de Fleury (à clapets) est équipé d'une passe à poissons et fait l'objet d'une gestion de ses clapets permettant la migration des aloses ; cependant, cet obstacle demeure difficile à franchir en période de basses ou de hautes eaux,

- en amont du seuil de Moussoulens, le seuil de Ferrioles n'est pas encore équipé, bien que la construction d'une passe ait été demandée au propriétaire (par les services de l'Etat, après avis du CSP) ; par ailleurs, plus en amont, la passe à poissons du seuil de Saint-Nazaire semble peu efficace pour l'alose.

D'autre part, sur le plan réglementaire, la modification de la limite de salure des eaux (actuellement en amont du seuil anti-sel) et la protection de la frayère de Moussoulens par arrêté de biotope proposées par le COGEPOMI (ANONYME, 1995), n'ont pas été réalisées.

Des études génétiques et écobiologiques ont été réalisées sur des aloses capturées sur ce fleuve de 1997 à 1999 (LE CORRE et al., 1998b). Elles montrent que cette population est très proche de celle du Rhône (LE CORRE, 1999).

Hérault (CRUZ, 1991)

Les études menées sur ce fleuve font apparaître que quelques aloses peuvent encore fréquenter ce cours d'eau, en aval d'Agde (LE CORRE, 1999). 
Peu d'actions initialement prévues dans le cadre du Contrat « Retour aux Sources » ont été réalisées.

La construction des passes du seuil d'Agde et du seuil du pont de Florensac, respectivement premier et troisième obstacles sur l'Hérault, était prévue pour l'été 1999. La passe à poissons du seuil d'Agde sera achevée à l'été 2000, mais la construction de la passe de Florensac n'a pas démarré.

Quant au deuxième obstacle sur le cours d'eau, le barrage de Bladier-Ricard (commune de Bessan), celui-ci a été équipé (travaux achevés en juin 1993).

En ce qui concerne le barrage de Saint-Thibéry (quatrième obstacle sur le cours d'eau), le propriétaire s'est vu dans l'obligation réglementaire de construire une passe à poissons. Celle-ci est cependant totalement inefficace pour tous les migrateurs.

Par ailleurs, la demande faite par le COGEPOMI d'extension du classement à migrateurs au titre de l'article L.232-6 (ANONYME, 1995) n'a pas été suivie d'effet.

Vidourle (BOURGOGNE, 1991) 1999).

Des aloses ont pu être capturés et étudiés en 1999 sur ce fleuve (LE CORRE,

En effet, quelques individus ont été observés sur le Vidourle au printemps quand le cours d'eau présente un débit d'appel suffisant. Dans le cas contraire, les aloses ne s'engagent pas très haut dans le Vidourle car le canal du Rhône à Sète croise le cours d'eau juste en amont de l'embouchure et présente en général un débit plus attractif.

A ce jour, aucune mesure spécifique à l'alose n'a été entreprise. Toutefois, le premier obstacle à la migration que constitue le barrage anti-sel de Terre-de-Port a été récemment détruit par des crues (1995 et 1996).

\section{Cours d'eau de la région Provence-Alpes-Côte d'Azur}

Les cours d'eau concernés sont le Var, l'Argens, le Gapeau et la Siagne.

II semble que le cours aval de l'Argens soit encore fréquenté par les aloses, même si les effectifs demeurent très faibles (LANGON, 1999a).

Actuellement, aucune mesure spécifique visant à faciliter la progression des poissons migrateurs n'a été entreprise, malgré les propositions de classement au titre de l'article L. 232-6 du Code Rural (arrêté ou décret) de ces cours d'eau faites par le COGEPOMI (ANONYME, 1995).

\section{Rivières corses (Figure 1)}

Seul le Tavignano a fait l'objet d'un état des lieux en 1999 (LANGON, 1999b). Celuici a permis d'observer et de capturer des aloses en aval de l'usine de Cardiccia (principal obstacle sur le cours d'eau), de repérer les zones potentielles de frai et de procéder à l'identification génétique du taxon (LE CORRE, 1999).

Lors de la construction de la micro-centrale de Cardiccia en 1992, une passe à poissons a été intégrée à l'ouvrage. Celle-ci s'avère inefficace pour l'alose, en particulier en raison de la hauteur de l'obstacle (11,5 mètres) (LANGON, 1999b).

Par ailleurs, la présence d'aloses a été signalée sur un autre cours d'eau corse, le Golo (LANGON, 1999b). 


\section{DISCUSSION-CONCLUSION}

Au terme des huit années d'études et de travaux engagés dans le cadre du Plan Migrateurs, la situation de l'alose sur le bassin du Rhône s'est incontestablement améliorée.

Le volet $A$ du programme de restauration prévoyait le retour de l'alose dans l'Ardèche. En termes qualitatifs, les mesures spécifiques mises en œuvre (éclusées à poissons, aménagement des cours d'eau, méthodes d'études et de suivi) ont permis d'atteindre cet objectif puisque la présence d'aloses dans l'Ardèche a été démontrée par des captures à la ligne et par des suivis de frayères. En termes quantitatifs, on ne dispose actuellement d'aucune estimation fiable du niveau d'abondance du stock et donc de son évolution depuis la mise en œuvre du Plan Migrateurs Rhône-Méditerranée.

Toutefois des actions prioritaires doivent être réalisées impérativement pour compléter les objectifs de départ du plan migrateurs (LEBEL et al., 1999) :

- mettre en place un tableau de bord des indicateurs du potentiel « migrateurs », des actions de suivi et d'aménagement, afin d'améliorer la lisibilité et l'utilisation des données, de disposer en permanence d'informations utiles à l'évaluation des actions, à la communication avec les partenaires du programme ou avec les élus, les pêcheurs ou le public,

- maximiser et évaluer le stock franchissant le barrage de Vallabrègues, à partir de la méthodologie mise au point en 1998 et sous réserve d'un minimum d'une cinquantaine de comptages visuels sur l'ensemble de la saison; la quantification du stock d'aloses franchissant le barrage de Vallabrègues est actuellement le seul moyen permettant, à moindre coût et sur le long terme, de disposer d'un indice d'abondance réel de la population et donc de suivre l'évolution du stock sur l'ensemble du bassin rhodanien; il n'en demeure pas moins que la mise en place d'éclusées spécifiques reste une méthode très limitée pour optimiser le franchissement de cet obstacle et de ceux situés plus en amont et que la méthode la plus efficace reste l'installation d'un ascenseur à poissons ; une méthode de comptage doit être envisagée pour l'ensemble des écluses situées en amont du barrage de Vallabrègues ; en particulier, des éclusées à poissons spécifiques sont réalisées à Avignon et Caderousse et devront être complétées par un système de contrôle de leur efficacité ; le matériel d'échosondage pourrait trouver là une utilisation,

- améliorer les possibilités de franchissement des obstacles, dès le cours aval (et notamment à Sauveterre), afin d'assurer le passage d'un potentiel reproducteur en cohérence avec le potentiel de production disponible en amont ; il apparaît alors essentiel de chiffrer les potentiels relatifs des différents axes et biefs, pour évaluer la faisabilité effective de telle ou telle partie du programme, et guider les choix de priorité dans les aménagements,

- intensifier le suivi de la pêche à la ligne, en prenant en compte plusieurs sites témoins (Vallabrègues aval usine et seuil de Beaucaire, Sauveterre, Cèze, voire Caderousse) sur lesquels des séries chronologiques de captures pourront être enregistrées ; cette étude sera complétée par la caractérisation biologique des individus capturés afin de savoir si la fraction du stock franchissant le barrage est identique à celle échantillonnée en aval les années précédentes,

- connaître le comportement du poisson en aval du barrage de Vallabrègues, afin notamment de déterminer le comportement des poissons face à l'obstacle (identification d'une zone de repli, temps d'attente, facteurs stimulant le passage de l'écluse) et leurs déplacements avant le franchissement de l'écluse, ainsi que les conditions d'attractivité de l'écluse; la méthode la plus performante est certainement le radiopistage comme l'ont 
montré MOSER et al. (2000) sur une écluse de navigation utilisée pour le franchissement de l'alose savoureuse (Alosa sapidissima) sur la rivière Cape Fear (Caroline du Nord, USA), même si elle implique des manipulations délicates sur les poissons ; un suivi par radiopistage permettrait également de suivre les poissons en amont de l'obstacle et donc d'identifier les zones nouvellement colonisées; elle s'avérerait enfin très utile en tant qu'étude préalable à la mise en place d'un ascenseur à poissons à Vallabrègues.

Des mesures complémentaires doivent également être envisagées à plus long terme et selon les moyens techniques et financiers mis à disposition sur les fleuves côtiers (LEBEL et al., 1999) :

- suivi des pêcheries professionnelle et amateur aux engins, en coordination avec le suivi national de la pêche aux engins du CSP : un suivi simple de la pêche au carrelet est nécessaire pour cerner précisément la fenêtre de migration et disposer d'indices complémentaires d'abondance du stock, même si ces indices pris isolément peuvent être biaisés ; étant située plus en aval que la pêcherie au carrelet, la pêche professionnelle constitue une référence en matière d'abondance annuelle et permet de comparer les niveaux de population d'une année sur l'autre et indépendamment des autres composantes de la pêcherie, même si le niveau de captures dépend fortement des facteurs hydro-climatiques,

- analyse des frayères ouvertes et potentielles : elle concerne d'abord l'inventaire et la typologie des frayères, pour relier les potentialités et le degré de fonctionnalité une fois la zone ouverte; de plus, il est nécessaire d'évaluer le degré de fonctionnalité des frayères, en suivant certains sites reconnus actifs lors des campagnes d'études précédentes ; toutes ces mesures, pour être efficaces, doivent s'accompagner d'une protection des zones essentiellement vis-à-vis des actions anthropiques (pollutions industrielles et thermiques, extractions de granulats, prélèvements d'eau).

Par ailleurs, de nombreuses actions et études restent à entreprendre pour améliorer encore la situation sur les fleuves côtiers, notamment pour les cours d'eau de la région Provence-Alpes-Côte d'Azur et de Corse.

Le volet B est actuellement en cours d'élaboration. II devra en outre impérativement porter sur :

- l'extension géographique du programme aloses aux fleuves côtiers méditerranéens et aux affluents de rive gauche du Rhône : le programme d'extension du plan migrateurs concerne déjà les départements de l'Aude, l'Hérault, le Gard et le Var (cours d'eau Aude, Hérault, Vidourle et Argens) ; il consiste essentiellement en un suivi de l'activité de pêche à la ligne grâce au même système de carnets que sur le Rhône,

- l'extension du programme à la restauration pour l'anguille : en effet, la restauration du stock d'aloses est la première réalisation d'un programme plus ambitieux qui vise à restaurer la fonctionnalité écologique du Rhône aval ; le programme de restauration de la population d'anguilles du bassin du Rhône a d'ores et déjà démarré en 1998 ; il fait suite au constat d'une très forte diminution des populations d'anguilles du bassin et comporte le suivi d'une station de piégeage installée par l'Université de Rennes et MRM dans le contre-canal de Vallabrègues, ainsi qu'une campagne d'alevinage en tête de bassin au niveau de six sites au sein de la Réserve de l'lle de la Platière (départements de l'Isère et de l'Ardèche) (FEUNTEUN et al., 2000) ; les méthodologies mises en œuvre et le choix des sites d'implantation constituent une première approche qui reste à améliorer et à compléter. 


\section{PARTENAIRES DU PROJET}

Dans le cadre du Plan Migrateurs Rhône-Méditerranée, les partenaires financiers de l'Association Migrateurs Rhône-Méditerranée sont le Ministère de l'Aménagement du Territoire et de l'Environnement, le Conseil Supérieur de la Pêche, l'Agence de l'Eau Rhône-Méditerranée-Corse, la Compagnie Nationale du Rhône, le Syndicat Intercommunal d'Aménagement du Bas-Gardon, le Syndicat Intercommunal Ardèche Claire, Electricité De France, Voies Navigables de France, l'Institution Interdépartementale du bassin Rhône-Saône, les Conseils Régionaux Languedoc-Roussillon, Provence-AlpesCôte d'Azur et Rhône-Alpes, les Conseils Généraux de l'Ardèche, des Bouches du Rhône, du Gard et du Vaucluse.

\section{BIBLIOGRAPHIE}

ALEXANDRINO P.J., 1996. Estudo de populaçoes de savel (Alosa alosa L.) e savelha (Alosa fallax, Lacépède). Analise da differenciaçao interspecifica, subestructuraçao e hibridaçao. Thèse de doctorat, Université de Porto. $180 \mathrm{p}$.

ALLARDI J., KEITH P., 1991. Atlas préliminaire des poissons d'eau douce de France. Campagne du Conseil de l'Europe "Comme un poisson dans l'eau ». Muséum National d'Histoire Naturelle, Conseil Supérieur de la Pêche, Cemagref, Ministère de l'Environnement. 45-48.

ALMACA C., 1988. Compte-Rendu de la Table Ronde: les poissons des îles de la Méditerranée Occidentale (Baléares, Corse, Sardaigne, Sicile). Bull. Ecol., t 19, 2-3.

ANONYME, 1991. Schéma de Vocation Piscicole du Rhône. DIREN Rhône-Alpes, Délégation de Bassin Rhône-Méditerranée-Corse. Service de la Navigation RhôneSaône. 5 volumes + plaquette + cartographie.

ANONYME, 1995. Plan de gestion des Poissons Migrateurs 1995-1999. Rapport de synthèse. Comité de Gestion des Poissons Migrateurs du Bassin RhôneMéditerranée-Corse. 33 p. + annexes.

ANONYME, 2000. Reconquête des axes de vie piscicole. Note Technique SDAGE №3. SDAGE Rhône-Méditerranée-Corse. Comité de Bassin RMC, Agence de l'Eau RMC, Préfet Coordonnateur de Bassin RMC, DIREN Rhône-Alpes Délégation de Bassin RMC. 84 p.

BARTHELEMY C., 1997. À l'abri des acacias en fleurs... les pêcheurs d'aloses du Rhône. Campagne d'études 1995. CNRS, DESMID. Association Migrateurs RhôneMéditerranée, $1995-n^{\circ} 6 / 8.102$ p. + annexes.

BARTHELEMY C., MAZENS Y., 1997. Suivi de la pêcherie en aval de l'usine-écluse de Vallabrègues. Estimation des captures des pêcheurs amateurs aux engins. Campagne d'études 1996, CNRS, DESMID. Association Migrateurs RhôneMéditerranée, $1996-n^{\circ} 6 / 8.42$ p. + annexes.

BECHU D., BLANCHET A., TABARDEL M., MENELLA J.Y., GENDRE L., 1995. Suivi de la remontée des aloses au niveau de l'aménagement de Vallabrègues. Influence des conditions de milieu. Campagne d'études 1994. Association Migrateurs RhôneMéditerranée, 1994-n`1/5. 32 p. + annexes.

BOURGOGNE M., 1991. Schéma Départemental de Vocation Piscicole et Halieutique du Gard. Documents Direction Départementale de l'Agriculture et de la Forêt, Fédération des Associations Agrées de Pêche et de Pisciculture.

CASSOU-LEINS F., CASSOU-LEINS J.J., 1981. Recherches sur la biologie et l'halieutique des migrateurs de la Garonne et principalement de l'alose Alosa alosa L. Thèse de Doctorat Institut National de Polytechnique de Toulouse, Sciences et Techniques en Production Animale, Ichtyologie Appliquée. 382 p.

CHAMPALBERT E., 1998. Inventaire des frayères potentielles d'aloses sur les bas et moyens Gardons. Campagne d'études 1996. EVI. Association Migrateurs RhôneMéditerranée, 1996-n`3/8. 54 p. + annexes. 
CHANGEUX T., PONT D., 1995. Current status of the riverine fishes of the french mediterranean basin. Biological Conservation, 72 , 137-158.

CHANGEUX T., ZYLBERBLAT M., 1993. Analyse des statistiques de pêche aux engins dans le bassin du Rhône. Deuxième partie : étude des captures. Bull. Fr. Pêche Piscic., 330, 271-294.

CRUZ O., 1991. Schéma Départemental de Vocation Piscicole et Halieutique de l'Hérault. Documents Direction Départementale de l'Agriculture et de la Forêt, Fédération des Associations Agrées de Pêche et de Pisciculture.

DESJAMES F., 1987. Schéma Départemental de Vocation Piscicole et Halieutique de l'Aude. Documents Direction Départementale de l'Agriculture et de la Forêt, Fédération des Associations Agrées de Pêche et de Pisciculture.

DOUCHEMENT C., 1981. Les aloses des fleuves français Alosa fallax (Lacépède, 1803) et Alosa alosa (Linné, 1758) - Biométrie, écobiologie : autonomie des populations. Thèse de l'Université du Languedoc (Montpellier). $377 \mathrm{p}$.

FEUNTEUN E., BOULLIER J., BRIAUDET P.E., LAFAILLE P., 2000. L'anguille du Rhône aval. Etude préalable à l'élaboration d'un protocole de suivi et de restauration. Laboratoire d'Evolution des Systèmes Naturels et Modifiés (Univ. de Rennes I), EDF Saint-Alban, DIREN Délégation de Bassin Rhône-Méditerranée-Corse.

GALLOIS C., 1947. L'aménagement du Vivarais par la Compagnie Nationale du Rhône. Ses incidences sur l'économie piscicole. Mesures tendant à les atténuer. Bull. Fr. Pêche Piscic., 146, 25-34.

GENDRE L., 1997a. Franchissement par les aloses de l'aménagement de BeaucaireVallabrègues. Campagne d'études 1994. Association Migrateurs RhôneMéditerranée, $1994-n^{\circ} 3 / 5.73$ p. + annexes.

GENDRE L., 1997b. Franchissement par les aloses de l'aménagement de BeaucaireVallabrègues. Campagne d'études 1995. Association Migrateurs RhôneMéditerranée, $1995-n^{\circ} 1 / 8.73$ p. + annexes.

GENDRE L., 1997c. Validation de la manœuvre d'abaissement partiel de porte amont. Campagne d'études 1995. Association Migrateurs Rhône-Méditerranée, 1995$\mathrm{n}^{\circ} 2 / 8.31 \mathrm{p}$.

GENDRE L., MENELLA J.Y., CORRAO B., 1997a. Suivi de la dévalaison des alosons. Campagne d'études 1995. Association Migrateurs Rhône-Méditerranée, 1995$n^{\circ} 3 / 8.28$ p. + annexes.

GENDRE L., MENELLA J. Y., DUMAS C., 1997b. Suivi de la reproduction de l'Alose feinte du Rhône. Campagne d'études 1995. Association Migrateurs Rhône-Méditerranée, 1995-n8/8. 46 p.

GENOUD D., 1996. Suivi des frayères potentielles d'aloses sur la rivière Ardèche en aval de Saint-Martin et le Rhône court-circuité. Campagne d'études 1996. FRAPNA Ardèche. Association Migrateurs Rhône-Méditerranée, 1996-n5/8. 19 p. + annexes.

GENOUD D., 1997. Recherche et caractérisation des frayères actives d'aloses sur le Rhône court-circuité entre Viviers et Pont-Saint-Esprit. Campagne d'études 1997. FRAPNA-Ardèche, Fédération de Pêche de l'Ardèche, C.O.R.A. Ardèche. Association Migrateurs Rhône-Méditerranée, 1997-n²/6. 40 p. + annexes.

GENOUD D., 1999a. Suivi de la pêche d'aloses en Ardèche. Rivière Ardèche et fleuve Rhône. Campagne d'études 1998. Fédération de Pêche de l'Ardèche, Conseil Supérieur de la Pêche. Association Migrateurs Rhône-Méditerranée, 1998-n4/7. 30 p. + annexes.

GENOUD D., 1999b. Suivi quantitatif de la frayère de Saint-Montan ; Rhône court-circuité de Donzère-Mondragon. Fédération de Pêche de l'Ardèche. Association Migrateurs Rhône-Méditerranée, 1999-n²/2. 23 p. + annexes.

GUILLARD J., THERRY F., COLON M., 1996. Etude de la migration des aloses. Utilisation des techniques acoustiques. Campagne d'études 1996. INRA de Thonon. Association Migrateurs Rhône-Méditerranée, 1996-n7/8. 42 p. + annexes.

GUILLARD J., COLON B., 1997. Etude de la migration des aloses. Utilisation des techniques acoustiques. Campagne d'études 1997. INRA Thonon. Association Migrateurs Rhône-Méditerranée, 1997-n5/6. 32 p. + annexes. 
GUILLARD J., VIDAL N., 1998. Etude de la migration des aloses. Utilisation des techniques acoustiques. Campagne d'études 1998. INRA Thonon. Association Migrateurs Rhône-Méditerranée, 1998-n5/7. 22 p. + annexes.

GUILLARD J., COLON B., THERRY F., VIDAL N., 1998. La migration des aloses (Alosa fallax rhodanensis) dans l'écluse de Beaucaire-Vallabrègues étudiée par un sondeur acoustique à faisceaux partagés : synthèse des campagnes 1996 à 1998. INRA Thonon. Association Migrateurs Rhône-Méditerranée, 1998-n6/7. 48 p.

JOLIMAITRE J.F., 1992. Franchissement par l'Alose feinte de l'aménagement de la chute de Vallabrègues. Etude du franchissement de l'écluse de navigation. Avant-projet de passe à poissons sur le seuil de Beaucaire. Mémoire de mastère. ENGREF, Conseil Supérieur de la Pêche Montpellier, FISH-PASS. 42 p.

JOUFFRE L., 1997. Etude de faisabilité d'une technique de comptage par échosondage des poissons transitant par l'écluse de Beaucaire-Vallabrègues. Campagne d'études 1995. Association Migrateurs Rhône-Méditerranée, 1995-n5/8. 33 p. + annexes.

KEINER A., 1985. Au fil de l'eau en pays méditerranéen. Aubanel, Avignon. 308 p.

KREITMANN L., 1932. Les grandes lignes de l'économie piscicole du bassin français du Rhône. Trav. Lab. Hydrobiol. Pisc. Grenoble, 27, 127-131.

LADET A., 1996. Inventaire des frayères potentielles d'aloses sur le bassin de l'Ardèche et le Rhône court-circuité. Campagne d'études 1995. FRAPNA. Association Migrateurs Rhône-Méditerranée, 1995-n4/8. 167 p.

LANGON M., 1999a. Etude des aloses des fleuves côtiers méditerranéens : l'Argens (été 1999). Association Migrateurs Rhône-Méditerranée, CSP Délégation de Montpellier, DIREN Délégation de Bassin RMC, ENSA Rennes. 21 p. + annexes.

LANGON M., 1999b. Etude des aloses des fleuves côtiers méditerranéens : le Tavignano (juin 1999). Association Migrateurs Rhône-Méditerranée, CSP Délégation de Montpellier, DIREN Délégation de Bassin RMC, ENSA Rennes. 19 p. + annexes.

LE CORRE M., BAGLINIĖRE J.L., SABATIÉ R., MENELLA J.Y., PONT D., 1996. Caractérisation morphologique et biologie de l'Alose feinte du Rhône. Campagne d'études 1996. Laboratoire Ecologie aquatique INRA Rennes, Laboratoire Halieutique ENSA Rennes, Laboratoire Ecologie des Systèmes Fluviaux CNRS Arles. Association Migrateurs Rhône-Méditerranée, 1996-nº/8.

LE CORRE M., MOULIN L., SABATIÉ R., BAGLINIĖRE J.L., 1997. La reproduction de l'Alose feinte du Rhône (Alosa fallax rhodanensis, Roule 1924) en aval du barrage de Vallabrègues. Campagne d'études 1997. Laboratoire Ecologie Aquatique INRA Rennes, Laboratoire Halieutique ENSA Rennes. Association Migrateurs RhôneMéditerranée, 1997-n²/6. $10 \mathrm{p}$.

LE CORRE M., ALEXANDRINO P., BAGLINIÈRE J.L., SABATIÉ R., $1998 a$. Caractérisation taxinomique et suivi biologique de la population d'Alose feinte du Rhône (Alosa fallax rhodanensis, Roule 1924). Campagne d'études 1997. Laboratoire Ecologie aquatique INRA Rennes, CECA-ICETA Université de Porto, Laboratoire Halieutique ENSA Rennes. Association Migrateurs RhôneMéditerranée, 1997-n6/6. $24 \mathrm{p}$.

LE CORRE M., BAGLINIĖRE J.L., SABATIÉ R., 1998b. Caractérisation écobiologique et génétique des populations d'aloses (Alosa sp.) présentes sur l'Aude. Campagne d'études 1998. Laboratoire Ecologie aquatique INRA Rennes, Laboratoire Halieutique ENSA Rennes. Association Migrateurs Rhône-Méditerranée, 1998$\mathrm{n}^{\circ} 7 / 7.15$ p. + annexes.

LE CORRE M., 1999. Eco-biologie et génétique des populations d'aloses (Alosa fallax spp.) de la Méditerranée française. Thèse, ENSA Rennes, $190 \mathrm{p}$.

LEBEL I., LE CORRE M., SABATIÉ R., BAGLINIĖRE J.L., 1997. Suivi de la migration et de la pêcherie d'aloses feintes du Rhône (Alosa fallax rhodanensis, Roule 1924) au niveau du barrage de Vallabrègues. Campagne d'études 1997. Laboratoire Ecologie Aquatique INRA Rennes, Laboratoire Halieutique ENSA Rennes. Association Migrateurs Rhône-Méditerranée, 1997-n¹/6. 74 p. 
LEBEL I., BAGLINIÈRE J.L., PORCHER J.P., SABATIÉ R., 1999. Bilan des actions et des études menées par l'Association Migrateurs Rhône-Méditerranée de 1991 à 1998. Laboratoire Ecologie aquatique INRA Rennes, Conseil Supérieur de la Pêche Délégation Régionale de Rennes. Association Migrateurs Rhône-Méditerranée, 1998-n ${ }^{\circ} 1 / 7.57$ p. + annexes.

LEBEL I., 1999. Suivi de la pêcherie d'Alose feinte du Rhône (Alosa fallax rhodanensis, Roule 1924) sur le bassin du Rhône. Campagne d'études 1999. Association Migrateurs Rhône-Méditerranée, 1999-n¹/2. 66 p.

LEGAULT A., 1993. Expertise pour l'accès des aloses et des anguilles aux affluents du Rhône. Inventaire des sites potentiels de frai de l'Alose. FISH-PASS. 97 p.

MAZENS Y., CORRAO B., 1997. Suivi de la migration des aloses au niveau de l'aménagement de Beaucaire-Vallabrègues. Campagne d'études 1996. Association Migrateurs Rhône-Méditerranée, 1996-n¹/8. 28 p. + annexes.

MAZENS Y., 1997. Suivi de la reproduction de l'Alose feinte du Rhône en aval du barrage de Beaucaire-Vallabrègues. Campagne d'études 1996. Association Migrateurs Rhône-Méditerranée, 1996-n²/8. 28 p. + annexes.

MENELLA J.Y., TABARDEL M., 1994. Suivi de la dévalaison des alosons à Caderousse. Définition d'un protocole d'évaluation des résultats. Campagne d'études 1993. Association Migrateurs Rhône-Méditerranée. 33 p.

MOSER M.L., DARAZSDI A.M., HALL J.R., 2000. Improvement passage efficiency of adult American shad at low elevation dams with navigation locks. North American Journal of Fisheries Management, 20, 376-385.

PATTEE E., 1988. Fish and their environment in large european river ecosystems: the Rhône. Sciences de l'eau, 7 (1), 35-74.

PINON M.P., 1998. Migration et caractérisation biologique de l'Alose du Rhône (Alosa fallax rhodanensis, Roule 1924). Suivi des pêcheries (1996-1998) et essai d'estimation du stock franchissant le barrage de Vallabrègues. Campagne d'études 1998. Laboratoire Ecologie Aquatique INRA Rennes, Laboratoire halieutique ENSA Rennes. Association Migrateurs Rhône-Méditerranée, 1998-n³/7. 42 p. + annexes.

PORCHER J.P., 1992. Poissons migrateurs : Contrat « Retour aux sources », propositions d'action. Conseil Supérieur de la Pêche, Ministère de l'Environnement. $169 \mathrm{p}$.

QUIGNARD J.P., 1978. Le Rhône et quelques problèmes concernant ses poissons ou histoire non naturelle de ce fleuve. Editions de la Sabranenque. $23 \mathrm{p}$.

RAMEYE L., KIENER A., SPILLMANN C.P., BIOUSSE J., 1976. Aspects de la biologie de l'Alose du Rhône. Pêche et difficultés croissantes de migrations. Bull. Fr. Pêche Piscic., 263, 51-76.

ROULE L., 1924. Les poissons des eaux douces de la France. Famille des Clupéidés. 6983.

ROURE F., 1997. Étude des zones potentielles de frai sur la Cèze. Campagne d'études 1996. ATOUT Environnement. Association Migrateurs Rhône-Méditerranée, 1996$\mathrm{n}^{\circ} 4 / 8.38$ p. + annexes.

ROURE F., VELEZ M., GARRIDOT S., 1997. Le retour des aloses sur la Cèze : Suivi de la frayère de substitution du Port de l'Ardoise, Laudun (30). Campagne d'études 1997. ATOUT Environnement. Association Migrateurs Rhône-Méditerranée, 1997- $n^{\circ} 3 / 6$. 38 p. + annexes.

ROURE F., SABATIER G., 1998. Le retour des aloses sur la Cèze : Suivi de l'efficacité de la passe à poissons de Codolet et des frayères potentielles en aval de Chusclan. Campagne d'études 1998. ATOUT Environnement. Association Migrateurs RhôneMéditerranée, 1998-n²/7. 34 p. + annexes.

RUCHON F., MENELLA J.Y., TABARDEL M., 1994. Reproductibilité du franchissement par les aloses des écluses de navigation sur le Bas-Rhône. Association Migrateurs Rhône-Méditerranée. $47 \mathrm{p}$.

TABARDEL M., MENELLA J.Y., GENDRE L., 1995. Suivi de la dévalaison des alosons à Sauveterre. Prospection de sites d'échantillonnage sur le Grand Rhône et le Petit Rhône. Campagne d'études 1994. Association Migrateurs Rhône-Méditerranée, 1994-nº/5. 44 p. 